\title{
Social bodies in virtual worlds: Intercorporeality in Esports
}

\author{
David Ekdahl $^{1}$ (D) Susanne Ravn ${ }^{1}$
}

Accepted: 1 March 2021/Published online: 27 April 2021

(C) The Author(s), under exclusive licence to Springer Nature B.V. 2021

\begin{abstract}
As screen-based virtual worlds have gradually begun facilitating more and more of our social interactions, some researchers have argued that the virtual worlds of these interactions do not allow for embodied social understanding. The aim of this article is to examine exactly the possibility of this by looking to esports practitioners' experiences of interacting with each other during performance. By engaging in an integration of qualitative research methodologies and phenomenology, we investigate the actual first-person experiences of interaction in the virtual worlds of the popular team-based esports practices Counter Strike: Global Offensive and League of Legends. Our analysis discloses how the practitioners' interactions essentially depend on intercorporeality - understood as a form of reciprocity of bodily intentionality between the players. This is an intercorporeality that is present throughout the players' performance, but which especially comes to the front when they engage in feinting. Acknowledging the intercorporeality integral to at least some esports practices helps fuzzying the sharp division between virtuality and embodied social understanding. Doing so highlights the fluidity of our embodied condition, and it raises interesting questions concerning the possibility of yet other forms of embodied sociality in a wider range of virtual formats in the world.
\end{abstract}

Keywords Intercorporeality $\cdot$ Esports $\cdot$ Intentionality $\cdot$ Embodiment $\cdot$ Virtuality $\cdot$ Sociality

\section{Introduction}

Screen-based virtual worlds ${ }^{1}$ facilitate more and more of our social interactions, and research into these kinds of interactions have seen an increased interest over the past

\footnotetext{
${ }^{1}$ This article focuses on typical, screen-based, virtual interactions set around more or less standard computer setups. Because VR, or Virtual Reality, brings with it its own new array of complexities, we here exclude it from our use of the concept of virtual worlds.
}

David Ekdahl

dekdahl@health.sdu.dk; david.edakhl@gmail.com

Extended author information available on the last page of the article 
two decades. Amongst this body of research, some authors have remained skeptical of virtual worlds' ability to facilitate bodily social cognition due to the virtual worlds broadly being viewed as disembodied or quasi-embodied (Dreyfus, 2009; Fuchs, 2014; see also Turkle, 2017; Dotson, 2017, Rosa, 2019, pp. 104, 123). The disembodied characteristic of virtual worlds is assumed in Dreyfus's (2009) seminal work on the internet. Here, he frames the discussion of the "Net's" and virtual worlds' current and future potential in terms of a clash between a skepticism towards the disembodying effect of virtual worlds and an optimism that virtual worlds might finally allow us to transcend our bodies in some form or other. Representing the side of the skeptic, Dreyfus echoes traditional phenomenological insights on embodiment, highlighting the subtle ways our living bodies are essential to social cognition (ibid., pp. 49-71); something we normally overlook because of the pre-reflective nature of our embodied condition (ibid., pp. 70-71). This subtle but essential role of the body is missing in virtual or tele-present spaces, Dreyfus argues, concluding that

[...] All this our body does so effortlessly, pervasively, and successfully that it is hardly noticed. That is why it is so easy to think that, thanks to telepresence, we could get along without it, and why it would, in fact, be impossible to do so. (ibid., p. 71)

Another similarly skeptical piece is Thomas Fuchs' article The Virtual Other (2014), wherein Fuchs explores the relationship between virtuality and empathy. Fuchs concludes that our sometimes seemingly real and empathetic relationships with others in virtual worlds are really more of a product of our own projection or imagination; they are "quasi"-empathetic encounters (ibid., p.155, 166) and not real or authentic the way a direct face-to-face encounter can be. This, he argues, is due in large part to the impossibility of engaging in real, felt bodily interactions in virtual worlds (ibid.)

While we are sympathetic to the central importance of our living bodies to direct social cognition, we nevertheless, in contrast to Dreyfus and Fuchs, wish to propose and discuss the existence of a domain of screen-based virtuality where direct, embodied social cognition is central to successful performance. We do this by looking to the reallife case of esports professionals, focusing on practitioners of the two games Counter Strike: Global Offensive and League of Legends. ${ }^{2}$ These esports practitioners make up a group of decidedly proficient users of a set of specialized virtual practices. Spending

\footnotetext{
${ }^{2}$ A note on the phenomena of 'physical esports' should be made: While traditional esports usually involve either a console or a personal computer setup, more whole-body forms of esports practices have begun to establish themselves around the world, for example in the form of virtual bicycle racing. In a time where Covid-19 is having a dramatic impact on the availability of sports events, taking one's physical talent into a virtual space might provide new opportunities. It also raises interesting questions regarding the possibility or impossibility of meaningful mergers between physical sports and virtual spaces, especially with regard to the relative lack of physicality in virtual spaces. In addition, whole-body exercise-games designed for the health benefits of esports practitioners have also begun drawing the attention of researchers (Martin-Niedecken \& Schättin, 2020). These areas of so-called 'physical esports' certainly afford more research - especially in terms of relative bodily immersion and sense of presence (Slater, 2009) compared to traditional, stationary esports. While we believe the embodied approach to sociality in esports we present here lay some of the groundwork for analyzing and understanding these practices as well, the domain of physical esports is a field in its own right, and falls outside the immediate scope of this article, including the professional backgrounds of the esports practitioners involved in this study, and as such we pursue it no further here. We are thankful to the anonymous reviewer who drew these phenomena to our attention.
} 
hours every day, typically from a young age, these practitioners refine and expand their virtual capabilities in the worlds of particular, competitive video games. Videogames have not escaped the critical lens of both Dreyfus and Fuchs, both raising critical points respectively vis-à-vis video games as part of their general criticism of virtuality as presented above. One such criticism from Dreyfus appeals to the non-reality of what is at stake in video games. Dreyfus argues that video games essentially only ever allow for a feeling of taking a risk without actually putting anything at risk. They "simulate serious commitments" (Dreyfus, 2009, p. 87) but not "serious commitments in the real world" (ibid., p. 87) While we wish to avoid the discussion of esports' place in the world of sports; for the professionals, the practice certainly entails many serious realworld commitments in terms of dedication, constant effort to improve, often being a personal livelihood, and often with an obligation to other people, for example teammates and fans. Serious players are not engaging with the games as simple entertainment or quasi risk-taking, and certainly not as a means of escapism from reality, but as a serious form of competition with something directly at stake - for many even as a profession and a form of excellence with their professional future on the line each time they compete. ${ }^{3}$

With a stronger phenomenological focus, Fuchs critically notes that video games do allow for a deceptive melding of body and computer, but he argues that gaming is nevertheless not really an embodied activity (Fuchs, 2014) as games offer only minimal resistance and no real elements of surprise compared to our normal bodily encounters with the world and others (ibid.). A gamer or an esports practitioner might here insist that virtual games are full of both surprises and resistances to be overcome, and in a later footnote, Fuchs (ibid.) responds to this insistence:

One could argue that components of surprise and resistance are also part of virtual games. However, here they belong to the preset frame of the game and, as such, they are expected by the player (ibid.).

Neither Dreyfus nor Fuchs includes descriptions from gamers in their analyses and accordingly do not build their argumentation on lived experiences of engaging with these virtual worlds in highly specialized ways. We believe one should. The aim of this article can thus be formulated as an investigation of esports practitioners' experiences of interacting other within the virtual worlds of selected games, accomplished by integrating qualitative research methods into the phenomenological analysis presented. On this interdisciplinary basis, we strive to further our understanding of virtualized perception and virtualized interactions and, not least, to critically discuss how intercorporeality is central for the mastery of these practices themselves. We present our understanding of this concept in more detail in the third section of this paper.

One might argue that analyzing esports practices as embodied can from the outset seem controversial as what the players are doing with their physical body (e.g. manipulating gaming peripherals) and what happens within the virtual world (e.g. avatarial movement) are not in a physical sense directly connected in the way that we directly engage with the material world and other people normally (Holt, 2016).

\footnotetext{
$\overline{3}$ Because of these factors, amongst others, it is very common for esports practitioners to suffer stress and deal with phases of burnout throughout their career (Smith et al., 2019).
} 
Whatever experiences of bodily immersion and interaction occur in the worlds of esports, must therefore instead be based on intellectual acts such as acts of evaluation about the virtual world. We do not subscribe to this insistence, and, as we have previously argued (Ekdahl \& Ravn, 2019), many esports practices do seem to involve elements of pre-reflective embodiment prior to intellectual acts. ${ }^{4}$ To insist on this is not to dismiss the importance of intellectual acts to esports practices; something especially central when learning how to move and act in the virtual spaces - as it is with any desktop system (Slater, 2009).

Similar ways of engaging with virtuality have been adopted by other researchers as well, who have likewise explored esports as an embodied phenomenon (Brenda, 2017; Hilvoorde \& Pot, 2016; Taylor \& Witkowski, 2010; Witkowski, 2012), while others have examined the connection between gaming and embodiment (Deng et al, 2013; Steed et al, 2016; Klevjer, 2012; Farrow \& Iacovides, 2013; Gee, 2008; Crick 2011; Black, 2015) as well as the possibility of online embodiment altogether (Osler, 2020; Tietz, 2015). What differentiates this paper from the above body of work is its inclusion of real-life data from virtual experts involved with the actual virtual practices explored and analyzed.

We here pursue our aim, first, by presenting an outline of esports and the two games involved before fleshing out the phenomenological notion of intercorporeality. Then follows an outline of the interdisciplinary methodology in use before we present the analysis of the two sets of virtual practices. Thus, we combine real-life descriptions with phenomenological analysis.

\section{Esports: An introduction}

In gaming - esports included - one can differentiate between two forms of interaction. One is player versus environment $(\mathrm{PvE})$, which refers to games or parts of games that pit human players against computer-controlled opposition in some form or other. Another is Player versus player (PvP), which refers to game practices where human players are pitted against each other. While elements of both are often present in esports games - but not always, as is the case with Counter Strike: Global Offensive - almost all esports practices most centrally revolve around their elements of PvP. This is important as it is less controversial ${ }^{5}$ to claim that pure PvE games offer less or little surprise and resistance of the sort Fuchs (2014) emphasizes given the predictability and limitations of computer-controlled opponents and environments. In the context of practices with a stricter PvP-focus - such as esports - where human players engage with and against each other, describing these practices as having a lack of resistance and surprise can be misleading.

A hallmark difference between computer-controlled opposition and humancontrolled opposition in video games and esports practice concerns exactly elements

\footnotetext{
${ }^{4}$ That esports is a fundamentally intellectual skillset is a claim that been made before. See for instance Jonasson and Thiborg (2010).

${ }^{5}$ Although, from the first author's own lifelong experience with gaming, it still seems that many - especially modern PvE-games - do offer meaningful forms of resistance and surprise. For one, because they often require fast-paced and coordinated teamwork with other human players, and, second, because of the increasingly complex computer-controlled opponents and environments found in video games.
} 
such as unpredictability and even spontaneity that humans can and do bring to the practices. Even though esports is delineated by certain rules and technological limitations - a "frame", to use Fuchs' term (ibid.) - the virtual practitioners nevertheless routinely develop and execute what we have previously dubbed "virtual Fosbury flops" (Ekdahl \& Ravn, 2019) - ways of innovating and surprising others within the frame of the competitive virtual setting. These typically expand what everyone involved with the game, often including the game designers themselves, thought was possible, changing the way the game is played and even developed henceforth (ibid.). One should therefore be careful to bar off the possibility of surprise and even resistance in these practices given their human element.

Esports, at its core, encompasses professionalized or organized competitive video gaming, ranging from top tier professional teams to local esports clubs and unions. Esports is surprisingly massive, with millions of people following their favorite players or teams excel at their favorite games in tournaments for prizes that today peak at over 15 million US dollars. These events take place both in some of the world's largest stadiums along with various live-streaming platforms available online (YouTube, Twitch, etc.). Esports events often draw in massive crowds both physically and virtually, at times outnumbering the amount of total spectators found in some of the biggest, traditional sports events (Dorsey, 2014; Pei, 2019). Of the millions of people around the world engaging with esports in one form or other, the professionals for this study are all involved with either one or both of the esports games Counter Strike: Global Offensive ('CS:GO') and League of Legends ('LoL').

Both CS:GO and LoL are based around personal gaming computers with both relying on similar gaming peripherals. Both CS:GO and LoL are team-based games with two teams of five players competing each round. Looking at the physical equipment and setup prior to the beginning of a game, one would be hard-pressed to tell the difference between the two esports practices, but the actual games do have several, crucial differences. Certain features of the games must therefore be described in order to lay the contextual ground for analyzing the practices. As we can only briefly sketch the game designs here, we encourage the reader to spend a moment familiarizing themselves with the perceptual layout of the different games online. ${ }^{6}$

\subsection{Cs:Go}

CS:GO is a first-person shooter (or FPS) where each player controls an avatar through whose eyes they perceive the virtual world. In CS:GO, basic movement involves both the keyboard and the mouse, with the keyboard generally controlling the lower half of the avatar's movement (running, "strafing", crouching, jumping, sneaking, etc.), and the mouse controlling upper body movement (turning the body and head, aiming, shooting, throwing grenades, etc.).

The objective of the game varies depending on whether one is playing as the 'terrorists' or the 'counter terrorists' with the players typically switching sides halfway through the total amount of rounds. The former begins in a position that generally requires some aggression to fulfill their goal - e.g. 'shoot the opponents and/or plant a bomb at one of two specific sites', the latter typically starting out in a more defensive

\footnotetext{
${ }^{6}$ See, for instance, this introduction to CS:GO or this introduction to LoL.
} 
position - e.g. 'prevent your opponents from planting the bomb either by shooting them or running out the clock'. Each professional player in CS:GO typically focuses on one of several available roles on a team, which, more than anything else, dictates how they move, act, communicate and what equipment they specialize using in-game.

\subsection{LoL}

LoL is a bird's-eye perspective, fantasy-based MOBA-game ${ }^{7}$ where two teams of five compete to wear down each other's home bases. These bases consist of several structures that provide advantages to a team, in order to finally destroy the innermost structure of the opposing team's base known as the nexus. Each player typically controls one character or avatar known as a champion. On the surface of it, the avatarial movements in LoL are simple: A player right-clicks the mouse whenever the cursor is located where he or she wants the champion to move on the map, and the champion begins moving towards the designated location. This simple dynamic, however, becomes increasingly complex and fast-paced the better the players involved, and proficient players will typically undertake such a relocation command several times per second throughout the game.

Furthermore, each of the many champions available are unique, possessing their own abilities that are unlocked and empowered gradually as the game progresses. These abilities synergize internally to the champion's intended play style as well as with the champion's intended role within a team composition. Similar to CS:GO, these roles are one of the most impactful dimensions of a player's playstyle, heavily dictating what a player is supposed to be doing, including how and where they move, communicate and equip themselves in-game.

\section{Intercorporeality}

In looking to esports interactions within the framework of intercorporeality, we lean on the writings of Merleau-Ponty (1964a, b, 2012), as he is a central phenomenological figure in terms of clearly and meaningfully disclosing the essential connection between embodiment and social understanding.

For a clearer sense of this, a good place to begin is the phenomenological concept of intentionality - the fundamental feature of consciousness to go beyond itself and be consciousness of something (e.g. physical objects - or virtual ones) in a particular manner (e.g. in perception, in memory, in fantasy). Here, Merleau-Ponty specifically focuses on the bodily mode of intentionality known as operative or functioning intentionality (Merleau-Ponty, 2012, p. 1xxxii), which he views as practical and basic to our existence- as the body's intentional entwinement with the world. According to Merleau-Ponty, it is this 'bodily intentionality', understood as our lived bodies' prereflective and practical relationship with world, that anchors us in the world at all (2012, pp. 140-141):

\footnotetext{
$\overline{{ }^{7} M O B A}$ is short for 'multiplayer online battle arena'.
} 
The motor experience of our body is not a particular case of knowledge; rather, it offers us a manner of reaching the world and the object, a "praktognosia," that must be recognized as original, and perhaps as originary. (ibid., p. 141).

Our bodily intentionality is therefore highly practical, and covers our "integrated suite of skills, capacities and habits" (Krueger, 2018) in a way where they can be appreciated as being direct parts of our body. These parts are not static aspects of our bodily existence, but plastic or moldable ones: Acquiring a new habit, such as mastering the piano (or a video game), is a modification of our bodily intentional grasp on the world, or body schema, as Merleau-Ponty (2012, p. 142) calls it. It is as embodied that we intentionally perceive and act in the world as a practical set of powers - as an "I can" (ibid., p. 155). One domain where we can appreciate the essential relationship between perception and body is in the world of sports. MerleauPonty himself provides such an example when describing the relationship between a football player and the field:

For the player in action the football field is not an 'object', that is, the ideal term which can give rise to a multiplicity of perspectival views and remain equivalent under its apparent transformations. It is pervaded with lines of force (the 'yard lines'; those which demarcate the penalty area) and articulated in sectors (for example, the 'openings' between the adversaries) which call for a certain mode of action and which initiate and guide the action as if the player were unaware of it. The field itself is not given to him, but present as the immanent term of his practical intentions; the player becomes one with it and feels the direction of the goal, for example just as immediately as the vertical and horizontal planes of his own body (Merleau-Ponty, 1967, p. 168).

For Merleau-Ponty, it is impossible to separate what we can do with the world from how we experience it, and our pre-reflective, practical relationship with the world as bodily intentional beings is an expression of the fact that perception and body are really two sides of the same coin.

It is this account of the body and its intentional correlation with the world that "provides the beginnings of a solution" (ibid. 364) to the difficulty of how to phenomenologically account for the experience of others. An important part of MerleauPonty's solution is that other's intentional behavior expresses the same bodily grasp of the world as my own body's intentional behavior does. As bodily intentional beings, we are able to recognize and understand each other as body-subjects because of this. Merleau-Ponty writes

I experience my body as the power for certain behaviors and for a certain world, and I am only given to myself as a certain hold upon the world. Now, it is precisely my body that perceives the other's body and finds there something of a miraculous extension of its own intentions, a familiar manner of handling the world (ibid. 370).

According to Merleau-Ponty, it is not merely because the body anchors us perceptually in the world that we can encounter other living bodies in that very world. For Merleau- 
Ponty, it is the aforementioned manner in which the body is anchored in the world that provides a familiarity with and thus an understanding of other living bodies as well. Merleau-Ponty thus ties our bodily intentional anchoring to the most primary form of social understanding we have: the experience of another living body. As is common to Merleau-Ponty's phenomenological descriptions, there is an explicit correlation or reciprocity in the encounter between living bodies. As Tanaka (2015) notes, this social understanding allows for a "grasping of the other's actions through one's own body" (ibid.) and, correlatively, a finding of “one's own possibility of action in another's body" (ibid.). That is to say, we encounter others as habitual seats of power- as living bodies with whom we can come to develop and share an implicit and practical understanding of the immediate and reciprocal meaning of our actions and movements in the world. We are in this way fundamentally intercorporeal beings (Merleau-Ponty, 1964a, pp. 170-172). Intercorporeality understood this way can therefore be appreciated as a form of reciprocity of bodily intentionality between embodied subjects - an entwinement of embodied, social understanding residing "beneath thought", as Merleau-Ponty notes (1964a, p. 170), and it is this account of intercorporeality that we follow here. ${ }^{8}$

\section{Method}

The investigation is grounded in an interdisciplinary methodology. In a two-tier fashion (Høffding \& Martiny, 2016) we first used qualitative research methods to generate empirical data and to identify central themes of esports players' practices and experiences. Second, we used these descriptions for a phenomenological analysis with an explicit focus on intercorporeality. The generation and first analytical phases of empirical data were thereby carried out according to validity and reliability criteria of qualitative research while the second phase or tier were carried out according to criteria of both "internal" and "external phenomenological consistency" (ibid.). Here, internal phenomenological consistency refers to the descriptions' ability to be made phenomenologically comprehensible, and external phenomenological consistency refers to the overall account's ability to relate to current theories of relevance to the phenomena. As the second author has discussed the background and promises of such interdisciplinary methodology in depth elsewhere (Ravn \& Hansen, 2013; Legrand \& Ravn, 2009; He and Ravn 2018; Ravn \& Høffding, 2017), the following sections focus on presenting the criteria for selection of informants, explicating the design and qualitive methods used when generating and analyzing data, explicating the way these data were used for the phenomenological analysis, and finally presenting an overview of participants involved with an orientation towards the standards suggested by Levitt et al. (2018).

\footnotetext{
${ }^{8}$ It should be noted that intercorporeality, as Fuchs (2016) has argued, can also more directly be appreciated as the bodily basis of our interaffective understanding of each other. A smile might carry joy, tears might express sadness, a scowl might convey anger because of our intimate familiarity with these phenomena as bodily beings. For our analysis, however, as there are no smiles, tears or scowls within the virtual worlds of CS:GO and LoL, we here focus primarily on movement- and action-based reciprocity of bodily intentionality of the ingame bodies of the players - and our use the concept is meant to reflect this focus. This is only intended as an analytical focus and should not be understood as a dismissal of the presence of affectivity nor interaffectivity in esports practice.
} 
The first author is himself familiar with both esports practices, having played several versions of CS:GO over the past twenty years at an amateur level as well as LoL for seven years, reaching the top one percept of amateur players in the latter. The first author has also followed esports culture for many years and continue to do so today. At several points during interviews, this familiarity allowed the researcher to discuss concrete examples from tournaments with the informants. Moreover, the first author's familiarity allowed for a close "alignment" of the researcher's second-person perspective with the first-person experiences of the informant. Ongoing discussions of the analysis with the second author ensured that he also kept a constructive distance to the descriptions of the informants (Legrand \& Ravn, 2009).

\subsection{Criteria for selection of informants}

The informants were selected based on three criteria: 1) A background in either LoL, CS:GO or both games. ${ }^{9}$ 2) Convincing capabilities with the game itself. ${ }^{10}$ 3) Training and/or coaching experiences in at least one of the two games. Criteria 3 was included as it was assumed that talented trainers and coaches are versed in describing their experiences in fine-tuned ways.

\subsection{Design}

The study involved a combination of short-term observations or pre-interviews preceding formal semi-structured interviews. Both the observations as well as pre-interview meetings were used to contextualize the interview situation beforehand and to finetune interview questions related to the interview topics so that questions were phrased on the premises of the present context. The observational periods lasted around two hours on average and were conducted at training sessions at the actual location of the gamers. Pre-interview meetings with the informants were conducted when observations were not possible and lasted between thirty and sixty minutes. In practical terms, observations and pre-interviews were important for preparing follow-up questions in relation to the questions in the interview guide. The formal interviews lasted on average around ninety minutes, with the longest interview lasting over two hours, and the shortest interview around one hour. These variations were due to the availability of the interviewees.

One pilot study was conducted early on wherein three esports students were interviewed following two observational periods of two hours. The pilot study was included in order to try out the combination of observations and interviews, especially with regard to how the observations could form a relevant background for adjusting the ways research questions were phrased and how follow up questions could be prepared.

\footnotetext{
${ }^{9}$ It should be noted that the two games encompass more than their respective esports scenes alone, with many different play modes, maps, and so on available in each game - and that we here focus strictly on the standard features of the two games that are adopted and used on the professional esports scene.

${ }^{10}$ These capabilities were judged in the context of the two games' ranking system and/or by whether or not the trainer had a background in semi-professional or professional esports.
} 


\subsection{The interview guides}

The interview guide was structured according to general practice-specific topics (Kvale, 2002/1994), and the prepared questions pertaining to these topics would vary a little between the two esports practices. As an example, questions pertaining to 'weapon use' were specific to CS:GO and questions pertaining to 'ability use' were specific to LoL. The overall topic of central relevance to this study ${ }^{11}$ was that of 'playing with others'. Exemplary questions and followup questions related to this subject included:

- What do you pay attention to during team fights?

- How do you sense the 'right' distance to an opponent?

- How do you experience other players' movements and actions?

- Do you read anything into these movements?

- Is there a difference between fighting an AI and fighting a human player?

- Do you experience an opposing avatar differently if the player is AFK? (Away From Keyboard)

- What is the role of feinting?

- How do you use 'baiting'?

\subsection{Transcription rules and transcription process}

The interviews were transcribed ad verbatim by the first author. They included diction, response tokens, non-verbal vocalizations, grammar, and game-specific slang, but excluded pronunciations, geo-ethnic accents and involuntary vocalizations (Oliver et al., 2005). In a few instances, not every interview was transcribed in full, as a few segments of some of the interviews carried little relevance to the project (e.g. a trainer talking about his vacation plans).

The interviews quoted in this article were all translated from Danish by the first author.

\subsection{Data analysis}

The data was analyzed on the condition of important concepts and themes indigenous to the practices themselves - also referred to as using an emic approach (Hammersley \& Atkinson, 2007, p. 194; Olive, 2014). The analytical identification of central emic themes took place in

\footnotetext{
${ }^{11}$ The data generated for this study is part of a larger data set which was generated over sixteen months and extends beyond the subject of this paper alone.
} 
an iterative process where the interviews were read through several times until there was an internal consistency in relation to the themes identified (Stake, 1995, p. 7).

The central emic themes identified included 'reading the other player's body language', 'dancing', 'experiencing the other players in terms of what they can do', 'understanding what an opponent sees and feels', 'baiting and lurking' and 'coordinating movements and actions'. From here, we moved into the second tier which makes up the actual phenomenological analysis with an explicit focus on intercorporeality (Ravn, 2016; He \& Ravn, 2017; Høffding \& Martiny, 2016; Ravn \& Høffding, 2017). This analysis is what unfolds in section 5 .

\subsection{The informants}

Contact was established with the informants in one of two ways: Five of the practitioners ('Bill', 'Conrad', 'Liam', 'Loke' and 'Lyn were contacted directly via email or instant messaging prior to the beginning of the project as part of a direct outreach out to several educational facilities in Denmark that had both a sports and an esports profile. Contact was established with the remaining seven esports practitioners via an outreach on a closed Facebook forum for Danish esports trainers and coaches. The names pertaining to the informants are pseudonyms. The twelve individual practitioners and their backgrounds were:

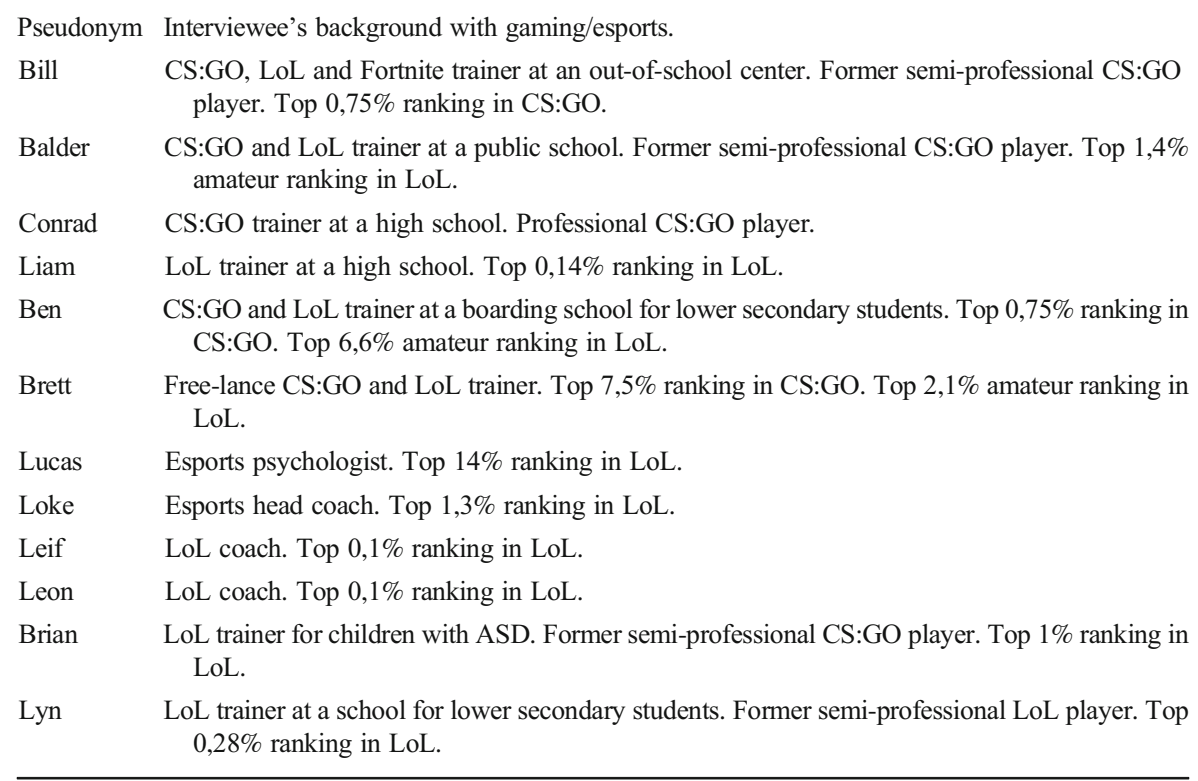

\section{Esports and intercorporeality}

In the following section, we analyze the role of intercorporeality within the virtual practices of CS:GO and LoL. We do this by analyzing different instantiations where 
reciprocity of bodily intentionality is pertinent to the two practices based on descriptions from the practitioners and of the practices. This culminates in our analysis of the in-game phenomenon of feinting, something that is common and essential to both esports practices at high levels.

\subsection{CS:GO}

As the players spawn, they are all locked in place for the first few seconds of the game with only the ability to purchase equipment and move their camera around. This being a first-person perspectival game where camera-body and avatar overlap (Klevjer, 2012) turning or moving one's avatar also entails moving one's perspective. Not much strategical or visual information is provided by these initial camera-movements as the players all already know the map intimately - "like their own apartment", one trainer noted - "in my bones", ${ }^{12}$ as another trainer formulated it. The concept of 'map' in esports, importantly, does not refer to an in-game map different or separate from the virtual world one is competing in, but rather refers to the overall game-world itself. The players do have access to an in-game map known as the 'mini-map'.

A few seconds later, the game announces that the round has begun. Each player, now able to move, and equipped with their chosen weapons and utilities, rushes towards a specific site on the map in coordination with their team, but not necessarily anywhere near them - virtually or physically. Before taking their first steps, each player has already holstered their gun(s) and brought out their tactical knife as one's running speed is dictated by the current weapon being held - and the knife, being the "lightest", allows the players to run the fastest; the lightness of the knife felt through the velocity of the avatar. Small but important shortcuts are taken to reduce the distance they need move, and a specific jumping technique known as 'bunny hopping' or 'bhopping' is executed at particular times by the players as they rush forward, speeding them up a little when they land. These forms of movement are habitual for the practitioners; they are simply the most optimal way to move around. The players' attention meanwhile fluctuates between a myriad of in-game factors. Based on his own experiences, Brett laid out the wide and demanding attention span of a good CS:GO player during performance:

A good player is attentive to many things in-game. First of all, he is attentive to the positioning of his teammates. Their place on the map. Where they are ingame. Which information are his teammates sharing? Which information does he himself pass on? He is aware of his own game; he is aware of the others' game. [...] He might be focused on his aim. He might be focused on his crosshair positioning. He might be focused on his own velocity ${ }^{13}$ : "How fast can I get from A to B" [locations on the maps known as 'Bombsite A' and 'Bombsite B']; "How quickly can I rotate between these sites?" Things like... pulling out your knife so you run faster. He is attentive to what utilities [e.g. grenades] to use. "Do I run

\footnotetext{
${ }^{12}$ Both translated from Danish, with the latter phrase being translated from "til hudløshed", literally meaning "skinlessly so".

${ }^{13}$ As we argued previously, forms of bodily agency and kinaesthesia are also present in these movements when the players navigate the virtual map (Ekdahl \& Ravn, 2019; see also Klevjer, 2012).
} 
with this strategy or that strategy?" "Do I use a smoke [grenade] or a Molotov cocktail?" "Do I use a flash bang?" It also depends on what weapons the others are buying... The players are paying attention to the in-game economy, which is a huge factor in everything. They are aware of the enemy team's in-game financial situation. With regard to the opponent, they are also attentive to what they are equipped with currently. What they purchased last round. And what are they anticipating the enemy team doing this round [emphasis by Brett himself] (Interview with Brett, lines 295-312).

As emphasized by Brett, a good player is first of all paying attention to his own team, as well as several dimensions directly related to his own team and the opposing team. In fact, the most emphasized ability to possess as a talented player, according to every CS:GO professional interviewed, was the ability to move, coordinate and communicate meaningfully and effectively with one's team, as well as keep close tabs on the opposing team's movements, positioning and even choice of weapons.

As a few more seconds of a round pass, the players inevitably enter territory where they anticipate enemies appearing. At this point, their guns are unholstered again and the team's various points of view spread out, every player ready on their individual trigger finger and microphone if an enemy should appear. Bill explained:

The first thing that happens in CS:GO is: You have a position you need to hold, or one you need to push, or something to do, and when you do this, you already have this sense of the different places the enemies are positioning themselves (Interview with Bill, lines 582-585).

If an enemy does appear, provided the weapon one is carrying harmonizes with the range to the opponent, the execution is swift and exact. A quick flick of the mouse readjusts the crosshair in the middle of the screen surgically towards the head of the now visible opponent. This is followed immediately by a single tap or two of the mouse - to keep the gun's recoil under control. If one is fast and precise enough, this takes the opponent out of the picture, eliminating his avatarial presence on the map for the time being.

Using any weapon in CS:GO proficiently entails an understanding of the different distances and tasks the weapons excel at within the virtual world (Ekdahl \& Ravn, 2019). Because of this, weapons are often aligned with the particular 'roles' as part of a team's overall strategy. A powerful sniper-rifle (known as an 'AWP'), for instance, while excellent at taking out opponents in long-range skirmishes and even shooting enemies through thick walls (ibid.), leaves one particularly vulnerable to opponents in close quarters - something immediately apparent to a talented AWP-player (known colloquially as an 'AWP'er' - something almost every team has at least one of). This understanding of the capabilities of the different weapons in the virtual world importantly carried with it a social element for the professionals: It cemented the need between the players to move in strict coordination and adjust their positioning in real-time so as to protect each other's weak spots while at the same time maximizing the potential of each player's equipment. Conrad noted that one's particular equipment would change "how your team moves and performs around you [in-game]" (Interview with Conrad, lines 332-333). 
Proficient CS:GO players needed to move and engage the opposing team as a cohesive unit across large, virtual distances if they wanted to win, and they therefore had to continuously paint a picture of the opponents' positioning over their voice communications, with the basic assumption and trust that everyone on the team immediately understood what the various information implied in terms of movement and positioning. The in-game coordination and communication exhibited by the professionals pointed to a constant awareness and understanding of each other's movement, responsibilities and capabilities during performance; of each player's ingame body and their weapon, as a "power for certain behaviors" (Merleau-Ponty, 2012, p. 370). "Your decisions and your plans also affect what the player next to you is going to do" [Conrad's emphasis] (Interview with Conrad, line 665), as Conrad noted. Bill elaborated on this in terms of the 'support' role on a CS:GO team:

Then you have your support player, who is likely running around behind his AWP'er. He sees things differently: "Where must I place myself to support my AWP'er? What grenades do I need to close off avenues? Based on where my AWP'er is positioning, what grenades do I need to carry in order to assist him in a get-away so as to not have him die? What angles must I be aware of so no one attacks him from behind? [...] [Bill's emphasis] (Interview with Bill, lines 362366).

A talented player was one whose movements and attention emerged in large part from a grasp of the meaning of the intentional movements and actions of the players around him, which itself entailed an understanding of one's own intentional movements and actions as phenomena that would reciprocally impact others' behavior around one's avatar. In this way, the players' team performance can be appreciated as relying on an in-game movement- and action-based reciprocity of bodily intentionality, a reciprocity of bodily intentionality that was a fundamental part of CS:GO, directing how players moved and positioned their avatars - often without explicit communication.

The players' joint movements and actions in-game can furthermore be appreciated as instantiations of collective intentionality, of individual players perceiving, moving and acting as part of a "We", and experiencing their own movements and actions as such. While each player controlled only one individual avatar, the players on a team needed to perceive, move and act as a collective intentional body if they were to be successful. We can make sense of this in-game collective intentionality as emerging from the bodily intentionality of the team coming to form an intercorporeal "system" where the meaning of the individual players' movements are inseparable from overall team (Walsh, 2020; Merleau-Ponty, 2012, p. 370).

Collective intentionality was characteristic of not just a single team's behavior, but also of the players' experience of the opposing team's behavior. In CS:GO, the ability to read the opponents' joint movement was a central and dynamic aspect of the practice that the players relied on pre-reflectively. The ability to do this was part of what the trainers called a good game sense:

A really talented player will anticipate anything. As I say, he will always be reading, reading what the opponents are doing, and, as I say, if the opponents are talented, they will constantly be trying to change up what they are doing so as to 
not be so easily readable. And then it becomes this back and forth - this mental game. [...] Those with a good game sense, it looks as if they are cheating because they are able to read the game so well. It seems as if they just know exactly where the opponents are [Bill's emphasis] (Interview with Bill, lines 330-332, 337-339).

The necessity of reading the opponents' movements and actions as part of a collective body has to do with the lack of visual access and time in which individual players on opposing teams can interact directly with each other in CS:GO. When talented, opposing players encounter each other in CS:GO, less than a second or two will typically pass before the players shoot each other down - leaving little time for sociality! "The first thing that happens in CS:GO when you see someone is... you have a millisecond to shoot them. The first thing that happens cannot be 'Heeey That's Peter over there!' It's 'There's an enemy. He needs to be shot'." (Interview with Bill; lines 577-578) This did not preclude intercorporeality between opposing players, only, instead of spending extended time interacting with individual opponents, the CS:GO professionals related to enemy movements and interactions as if each enemy player encountered also themselves moved and acted as part of a single, collective intentional body. As Bill noted "A good team plays against other teams" [Bill's emphasis] (Interview with Bill; line 563). Trainer Ben, elaborated:

Well, how are [the enemies] positioning? Are they playing aggressively? Are they playing passively? If you wait a minute before doing anything during an eco-round, ${ }^{14}$ well, are they coming to you then? "Okay, they are." Well, then we find them to be impatient. And we can use this to our advantage (Interview with Ben, lines 194-197).

Based on brief encounters with the opposing players along with verbal reports of their location and movement, players could in this way experience an enemy team as "stressed", "impatient" or "incoherent", and a talented players would, as Bill formulated it, understand when to be "passive" and when to be "aggressive" (Interview with Bill, lines 446-447). What any one opponent's intentional movements and actions signified was experienced as belonging to the collective intentional body of the enemy team, and this understanding was itself based on the players' bodily intentional familiarity with the "manner" (Merleau-Ponty, 2012, p. 141) of the virtual movements and actions in-game. Reciprocally, this intercorporeal understanding of an opposing team's collective intentional movements and actions was something integral to outmaneuvering one's opponents and thus surprising them based on how the players themselves 'revealed' their own intentions through their positioning and movement. We return to this in greater detail when discussing the phenomenon of virtual feinting.

\footnotetext{
${ }^{14}$ An eco-round, or 'economy round', is effectively a round in CS:GO where a team refrains from using too much of their currently owned cash in order to have a larger cash pool saved for the next round, allowing for a stronger impact at that point. These rounds are typically viewed by the team as more acceptable to lose due to the inferior equipment and lower amount of cash invested.
} 


\subsection{LoL}

In LoL, not unlike CS:GO, movement is hampered at the start of a round. Each team of five is locked inside a small zone within their own base with only the ability to purchase starting equipment and move around a tiny, encapsulated area. One will nevertheless often see the players moving jitteringly around within this starting zone. Some players do this to entertain themselves or pump themselves up, others to adjust the settings of their gaming peripherals and then others, according to the interviewees, use this time to get a feel for their own avatar's movement and ability to turn around. As indicated in the introduction to LoL, the biggest perceptual difference between LoL and CS:GO concerns the separation of camera-body and avatar in LoL. A player can move their perspective around on the entire map regardless of where their avatar is, but whatever in-game changes they want to directly incur will always be enacted through their champion and its abilities. This entails that a player's action-capabilities (what the champion can do) in the world and the player's perspective (the hovering camera) do not neatly and automatically overlap when performing.

During performance, LoL players, like CS:GO players, were constantly in touch, continuously using the voice chat to update their teammates on the activities of the enemy team - in case their teammates' attention was elsewhere. This was especially prominent during team fights and smaller skirmishes, where the players would clash together in complete pixelated carnage. Anyone who has listened to the live commentators or a professional team's voice communication during a LoL team fight or smaller skirmish will have a sense of just how much coordinated and complex movement and action is compounded into a few seconds. During these encounters, each player involved needs to have an immediate sense of who to avoid, who to stay close to, which specific abilities they need to anticipate, how everyone is moving, if any one opponent is particularly vulnerable, and how the overall power balance of a team fight is shifting in real time so as to know exactly when to retreat and when to push on - including an openness towards potentially innovative moves and actions ingame (Ekdahl \& Ravn, 2019). These interactions had immediate social ramifications for the entire team and were understood and executed as having such. A proficient player did not play or even perceive as an isolated champion on a team but rather had to move and play with a constant awareness of being part of the team, much like in CS:GO. In terms of team coordination, we thus find instances of collective intentional behavior in LoL similar to CS:GO, with the addendum of one's very visual field actually being directly shared by the rest of the team.

Because of LoL's bird's-eye perspective, the game also allowed for much more direct movement-based forms of interactions between opposing players compared to CS:GO - despite CS:GO being a first person-perspectival game with an intuitively closer perceptual and kinaesthetic semblance to everyday embodiment (Klevjer, 2012). The bird's eye perspective and distinction between camera body and avatar in LoL allowed opposing players to perceive each other from high above, moving and interacting around near each other for extended periods of time - often on a one-on-one basis. Players would spend several minutes moving visibly back and forth near each other, harassing each 
other by landing blows and abilities while also trying to avoid being similarly harassed by the opponent. This possibility for extended interactions with opponents was much more prominent than in CS:GO, where an encounter with a directly visible enemy was essentially a short-lived interaction before someone received a virtual bullet to the face.

In LoL-interactions, the encounter with other players was informed by the abilities of the players' champions as different champions in LoL are able to do very different things in the virtual world and move very differently (Ekdahl \& Ravn, 2019). This had an immediate influence on how and when the players moved around and interacted with their opponents, including what they paid attention to. Liam noted:

If [the opponent] is playing a champion that wants to jump in your face, for example, then you are typically looking for moments when he moves closer to you and reaches the distance needed where he has to jump you, right? This is why it's important to grasp the proper distance to your opponent, and things like that. Because then you will be able to see when he is faking, and when he really means it. When he is really looking for it. [...] Often, then, it's standard practice of the [early phase of the game] to fire off one or two [attacks towards your opponent] in order to read his patterns of movement [Liam's emphasis] (Interview with Liam, lines 361-370).

Grasping "the proper distance", reading opponents" "patterns of movement", and understanding when a player "really means it", as Liam notes, was not something done by looking up the units of distance pertaining to the implicated champion, but had to do with a practical familiarity with the champions themselves. The LoL-professionals agreed that a good player, while specializing in only a few champions, needed to have played all the different available champions in-game in order to maintain a proper sense of each champion's capabilities and ideal movement styles. This was important to the players' sense of where and how to move in response to or conjunction with other players' movements. LoL-professional Lyn described the way avatars moved near each other in LoL thusly:

It's all a dance. Including the [early] phase of the game. If I take one step forward, my opponent will take a step back. If I take a step to the right, my opponent will take a step to the left. And when we get into team fights, then we see the exact same thing happening. Then you look for who frightens you, and if he takes two steps forward, then we take three steps back. If he isn't present, we will take two steps forward - maybe - provided he is further back or has used a particular ability. Then it becomes a dance (First of two interviews with Lyn, lines 967971).

This in-game dance has the appearance of opposing avatars 'stepping' precipitously back and forth several times per second near each other in the game's two-dimensional space, smoothly fluctuating between a safe distance and the distance needed to be able to hit the opponent(s). To an untrained spectator, these individual rapid movements seem random - even erratic - but to the 
proficient practitioners, this 'dancing' was something highly expressive in terms of bodily intentionality. Strategically, this was important to the informants because how one moved was seen as directly entwined with the movements of the nearby opponent, which, if these suddenly underwent small changes in style, were sources of information, potentially signaling the beginning of anything between an ambush to a smaller or bigger skirmish. One LoL-trainer explained:

There is something in these... movements, where you can read a whole lot. I guess that is how it is. I never thought about it like that, but that is really how it is. Right, right - that's what one does! And it is actually from that, that one makes his decisions along the way! ${ }^{15}$ (Interview with Balder, lines 168-172)

We can appreciate Balder's descriptions of these movements as something intercorporeal, that is, as a reciprocity of bodily intentionality based on Balder's own close practical "familiarity" (Merleau-Ponty, 2012, p. 370) with the manner of movements and actions specific to the virtual space (ibid., p. 141).

Notably, individual practitioners each moved and acted slightly differently - something important in terms of reading their "patterns of movements" as Lyn formulated it. This speaks to degree of individuality in terms of movement style in LoL, no doubt facilitated by the game's more visually accessible forms of interactions. For instance, as a LoL-round progressed and the map eventually became more of an open battlefield between the two teams, the players on a team would begin encountering other individual opponents that they had not yet 'danced' with. Here, despite the minimal forms of available motility of the game's "frame" (Fuchs, 2014), the interviewees noted that they would not feel as attuned to these new opponents' behavior as they did with the opponent(s) they had been 'dancing' with previously. They had seemingly developed a bodily familiarity not just with a generic LoL avatar, but with an individual one. This speaks to a virtual form of "intercorporeal memory" as Fuchs (2016) has described it - wherein individual LoL practitioners could cultivate a bodily familiarity with the specific style of individual opponents' reciprocal intentional behavior.

We see that in both CS:GO and LoL, the interactions between the players can be as instantiations of reciprocity of bodily intentionality, that is, of intercorporeality. To further cement the centrality of intercorporeality in these practices, we now turn to a phenomenon central to both CS:GO and LoL at a professional level: that of feinting.

\subsection{Feinting in CS:GO and LoL}

The phenomenon of feinting in competitive sports has drawn the attention of philosophy, sports sciences and psychology alike (Aggerholm et al., 2011; Güldenpenning et al., 2017; Lynch et al., 2019; Park et al., 2019; Sebanz \& Schiffrar, 2009). We here understand feinting to be form of competitive deception based in large part around bodily intentional movements and actions. In competitive sports, feinting is done for

\footnotetext{
${ }^{15}$ Balder's own sense of surprise at this was striking to the first author. Balder had seemingly related to his opponents' movements this way throughout his life as an esports practitioner and trainer without ever putting it into words; without reflecting consciously on it.
} 
strategic reasons, and typically with the purpose of deceiving opponents regarding one's intentions. Feinting can thus be appreciated as an intercorporeal phenomenon, relying heavily on the reciprocity of bodily intentionality between a plurality of embodied subjects directly trying to make sense of each other's movements and actions in real-time. It is because of the movement- and action-based reciprocity of feinting that the phenomenon is relevant to the final part of our analysis.

Feinting turned out to be a central feature of CS:GO expertise, as well as a phenomenon that depended heavily on the primacy of the aforementioned bodily collective intentionality between the CS:GO players. Its prominence was brought up during the interview with Conrad, whose role on the team was that of a 'lurker'. As he described it, his job on the team was essentially to be a loose end or a wildcard in the eyes and minds of the opposing team. In order to do this successfully, he had to be deceptive in how he moved and how and when he chose to reveal himself to the opposing players round by round, understanding full well that his appearance would immediately be communicated amongst the opponents and become part of their understanding of his team. In his words:

There are a lot of in-depth things you have to consider and communicate - such as... you need to have a massive insight into what your opponents are seeing and feeling once you start throwing your grenades around on the map. You need to be able to conjure up an image for your opponents of "Hey, I am building a pressure over here." Then you begin to incept something so deep [into the enemy team]... Let's say we do one round, where I make a successful lurk. And a successful lurk is one where one basically makes it behind enemy lines. And now - in the following round - because you may have shot an opponent or two, they are now incredibly paranoid: "We have to make sure he does not break through our lines again." Then, in that round, you start making these mind games. You begin playing with the idea that maybe the more powerful play right now is not to use your role as a lurker, but instead fake that you are going to. This is something you can do at several places on the map. And, again, you can twist it around like that. So, you must be good at navigating your opponent's rotation with this role, and, yeah, you need to develop a sense of "What are my opponents feeling?" "What are my opponents seeing?" [...] [Conrad's emphasis] (Interview with Conrad, lines 126-138).

In this way, a successful lurk depended on an understanding of the opposing team relating to the lurker's team as collectively intentional in the way described previously, when, in fact, the lurker would often play against that very penchant of collectivity. A successful lurk depended on Conrad having a sense of what the opposing team was feeling ${ }^{16}$ and seeing in order to navigate their understanding of his behavior, and thus how to deceive them. Later on, when expanding on how he managed to do this and how much attention he paid to the opponents' perceptions and emotions, Conrad noted:

\footnotetext{
$\overline{16}$ This indicates not just a reciprocity of bodily intentionality between Conrad and his opponents, but also an openness to a more specifically affective form of intentionality on the enemy team. While we do not wish to expand on this here, we have already noted that affectivity has also been closely connected to intercorporeality (Fuchs, 2016).
} 
Conrad: When I am performing, I do not think about it. Or, rather, I do not think about that I think about it - if that makes sense?

Interviewer: You do not think about that you think about it? Yes, that makes sense.

Conrad: It is just something I do after having played for this long. It's something that occurs naturally - it is the best way to do it. (Interview with Conrad, lines 431-435).

Conrad's understanding of his opponents and their experiences was thus not something explicit, but a pre-reflective awareness throughout a match that he had come to master by excelling at the game, and something he as a lurker relied on especially to be successful. We can make sense of Conrad's deceptions as dependent on the reciprocity of bodily intentionality between both teams we have already described, with Conrad's goal being to abuse that very structure by moving and acting against the opposing team's understanding of him and his team's collective intentional movements and actions.

Turning to LoL, one finds, particularly during the moments where players are jitteringly moving around near each other at prolonged periods of time, a constant presence of smaller and bigger attempts at feinting. When two opposing players are dancing back and forth with each other, the opponent might suddenly appear braver or less cautious in his in-game movement-style. If the other player picks up on this stylistic change, it will immediately be an issue to him or her. Perhaps the opponent is feinting courage to gain more dominance, or perhaps the bravery is really eagerness because an ally of the opponent is already prowling nearby, which is causing the first opponent to move and act braver as he gets ready to pounce on what he believes is an unsuspecting player.

Either way, the player might immediately react in some way to the change in movement style, e.g. by getting to a safe distance. Alternatively, if he is aware of the impact of his movements on the opponent's behavior, he might instead deceptively suppress his immediate impulse to seek safety, knowing that his opponent will also reciprocally be sensitive to this intentional behavior. If he or she reacts with apparent cautiousness in response to the opponent's increased courage - for instance, by backing off - the opponent will likely sense that their planned ambush has been revealed, and they will simply have to call it off for now so no time is wasted waiting around for the trap to fail. Instead, a talented player can 'string along' an enemy bluff for an extended period of time, staying at just the "proper" distance as discussed previously, in order to prevent the possibility of the ambush but nevertheless not giving the impression of knowing that the ambush exists, wasting both opponents' precious time. In this context, the interviewed LoL practitioners frequently noted the importance of seeing "through" opponents' deceptive movements. Lyn explained how LoL players, when moving jitteringly in front of one another, would:

[...] read each other's body language. Each other's random movement. Because they are actually not all that random. Subconsciously, one has an idea of what one is doing. And it's very hard to hide your intentions from another person as talented as you (First of two interviews with Lyn, lines 624-626). 
The ability to understand the intentional movements and actions of an opponent's feinting was connected the players' level of proficiency with the game, as well as requiring prolonged bodily reciprocal exposure to the style of the individual opponent(s) as discussed previously. The former of these two aspects echoes an element of feinting in traditional sports already indicated in previous research, namely that proficiency with a movement practice sharpens a practitioner's ability to perceive feinted movements as deceptive (Güldenpenning et al., 2017; Lynch et al., 2019; Park et al., 2019; Sebanz \& Schiffrar, 2009). On the flip side of this, according to the LoLpractitioners interviewed, a time this sharpness proved disadvantageous was when they were facing off against vastly inferior opponents who had little to no understanding of feinting even being a thing within the virtual world. These novices lacked the "familiar manner of handling the world" (Merleau-Ponty, 2012, p. 370) central to intercorporeality; something that made misleading them through deceptive forms of bodily intentional behavior virtually impossible.

All in all, while feinting in the virtual worlds of CS:GO and LoL took on different forms, the overall purpose was still to mask or mislead, be it as a team or a player, in ways that manipulated the opponents' immediate, bodily familiarity with the intercorporeal manner of the in-game movements and actions.

\section{Virtual intercorporeality}

Carefully describing and analyzing esports professionals' virtual practices and experiences, we have shown that drawing a sharp, dividing line between embodiment and (screen-based) virtuality is problematic as certain virtual practices depend on embodiment in the form of bodily intentional reciprocity between the practitioners involved. These virtual practices thus challenge the more encompassing disembodied accounts of virtuality as presented by Dreyfus (2009) and Fuchs (2014). We do not show that all virtual practices are necessarily intercorporeal, or even embodied, but we do show that certain virtual practices can be intercorporeal albeit each in their own manner. On this point, it is important to keep in mind the demarcating effect of any virtual practice, esports included. As an example, when discussing her awareness of the emotional states of opponents when performing, Lyn noted:

The human emotions are there, but [the other player] also has a life on the side, and maybe he is hungry or something. You do not think about these things. It's only the things that are relevant to my game ${ }^{17}$ (Second of two interviews with Lyn, lines 854-855).

Technology itself can structure what is possible in terms of social understanding. As Ihde (1979, pp. 9-10, pp. 21-26) has noted, technology-based mediation involves an amplification/reduction-structure; it allows for or enhances certain forms of 'mediation', but it also delineates that very mediation, and we should remain sensitive to both

\footnotetext{
${ }^{17}$ Due to the scope of this paper, we have not delved deeper into the connection between affectivity and sociality in esports performance. The quote from Lyn is intended only to underscore a limiting dimension of virtual intercorporeality of the sort explored in this paper.
} 
aspects before embracing the intercorporeal potential of a virtual medium. We find a similar insight in the writings of Leder (1990 pp. 181-182), who notes that every technological extension bears with it its own telic inclination towards certain "modified forms of interaction", whereby the technologies with which we extend our bodily being also impacts and structures the very activities themselves. Investigating esports professionals as a set of 'virtual experts', examined through the lens of phenomenology integrated with qualitative research methodologies, shows us what is possible but not what is necessary. By typifying an 'extreme case' of the sort discussed by Flyvbjerg (2006), in this case of virtual embodiment and intercorporeality, esports professionals show us not what must be the case, but what can be the case.

The insights generated here are to the benefit of anyone interested in virtual embodiment, as they provide evidence for the possibility of intercorporeality in virtual spaces far removed from what we might normally consider bodily. They raise interesting questions regarding the possibility of other forms of bodily sociality in virtual formats far less professionalized and competitive than esports, including, as an example, the virtual spaces of social media. Moreover, the insights generated here are of relevance to anyone involved with esports practice itself, including esports trainers, who have so far lacked a precise vocabulary to describe and engage with the kinds of bodily interactivity and reciprocity at stake in their practices.

Acknowledgements We are grateful to the many esports professionals that dedicated time and space to participate in the overall research project. We also wish to extend our thanks to The Danish Ministry of Culture for their funding of the overall project. We are thankful to Giovanna Colombetti, Lucy Osler and Joel Krueger for their feedback and comments on previous versions of this paper.

Code availability Not applicable.

Funding This research was funded by the University of Southern Denmark '(Department of Sports Science and Clinical Biomechanics' and 'Department for the Study of Culture') and 'The Danish Ministry of Culture'.

Data availability Not applicable.

\section{Declarations}

Conflicts of interest/competing interests Not applicable.

\section{References}

Aggerholm, K., Jespersen, E., \& Ronglan, L. T. (2011). Falling for the feint - an existential investigation of a creative performance in high-level football. Sports, Ethics and Philosophy, 5(3), 343-358.

Black, D. (2015). Why can I see my avatar? Embodied visual engagement in the third-person video game. Games and Culture, 12(2), 179-199.

Brenda, H. K. S. (2017). Spectating the rift: A study into eSports spectatorship. eSports Yearbook, pp. 9-35.

Crick, T. (2011). The game body: Toward a phenomenology of contemporary video gaming. Games and Culture 6(3):259-269.

Deng, S., Chang, J., Zhang, J. J. (2013). A survey of Haptics in serious gaming. Games and Learning Alliance, pp. 130-144. 
Dorsey, P. (2014). 'League of Legends' ratings top NBA finals, World Series clinchers. [Online] Available at: https:/www.espn.com/espn/story/_page/instantawesome-leagueoflegends-141201/league-legendschampionships-watched-more-people-nba-finals-world-series-clinchers

Dotson, T. (2017). Technically together: Reconstructing community in a networked world. MIT Press.

Dreyfus, H. (2009). On the internet (2nd ed.). Routledge.

Ekdahl, D., \& Ravn, S. (2019). Embodied involvement in virtual worlds: The case of eSports practitioners. Sport, Ethics and Philosophy, 13(2), 132-144.

Farrow, R. \& Iacovides, I. (2013) Gaming and the limits of digital embodiment. Philosophy \& Technology.

Flyvbjerg, B. (2006). Five misunderstandings about case-study research. Qualitative Inquiry.

Fuchs, T. (2014). The virtual other: Empathy in the age of Virtuality. Journal of Consciousness Studies, 5(2), $152-173$.

Fuchs, T. (2016). Intercorporeality and Interaffectivity. In: Intercorporeality: Emerging Socialities in Interaction. S.1.:Oxford University press.

Gee, J. P. (2008). Video Games and Embodiment. Games and Culture, 3(3-4), 253-263.

Güldenpenning, I., Kunde, W., Weigelt, M., 2017. How to trick your opponent: A review article on deceptive actions in interactive sports. Frontiers in Psychology, Volume 8.

Hammersley, M., \& Atkinson, P. (2007). Etnography: Principles in practice. Routledge.

He, J., \& Ravn, S. (2017). Sharing the dance - On the reciprocity of movement. Phenomenology and the Cognitive Sciences, 17(1).

He, J., \& Ravn, S. (2018). Sharing the dance - on the reciprocity of movement in the case of elite sports dancers. Phenomenology and the Cognitive Sciences 17(1):99-116

Hilvoorde, I. v. \& Pot, N., 2016. Embodiment and fundamental motor skills in eSports. Sport, Ethics and Philosophy, pp. 14-27.

Høffding, S., \& Martiny, K. M. (2016). Framing a phenomenological interview: What, why and how. Phenomenology and the Cognitive Sciences, 15, 539-564.

Holt, J. (2016). Virtual domains for sports and games. Sports, Ethics and Philosophy, 10(1), 1-9.

Ihde, D. (1979). Technics and praxis. Pallas Paperbacks.

Jonasson, K., \& Thiborg, J. (2010). Electronic sport and its impact on future sport. Sport in Society, 13(2), 287-299.

Klevjer, R. (2012). Enter the avatar: The phenomenology of prosthetic Telepresence in computer games. In The Philosophy of Computer Games (pp. 17-39). Springer.

Krueger, J. (2018). Intentionality. In: G. Stanghellini, et al. eds. Oxford Handbook of Phenomenological Psychopathology. S.1.:S.n.

Kvale, S. (2002/1994). En introduktion til det kvalitative forskningsinterview [an introduction to the qualitative research interview]. Hans Reitzels Forlag.

Leder, D. (1990). The absent body. University of Chicago Press.

Legrand, D., \& Ravn, S. (2009). Perceiving subjectivity in bodily movement: The case of dancers. Phenomenology and the Cognitive Sciences, 8, 389-408.

Levitt, H. M., Bamberg, M., Creswell, J. W., Frost, D. M., Josselson, R., \& Suárez-Orozco, C. (2018). Journal article reporting standards for qualitative primary, qualitative meta-analytic, and mixed methods research in psychology: The APA publications and communications board task force report. American Psychologist, 73(1), 26-46.

Lynch, S. D., Olivier, A.-H., Bideau, B., \& Kulpa, R. (2019). Detection of deceptive motions in rugby from visual motion cues. PLoS One, 14(9), e0220878.

Martin-Niedecken, A. L., \& Schättin, A., 2020. Let the Body'n'Brain games begin: Toward innovative training approaches in eSports athletes. Frontier in Psychology, Volume 11.

Merleau-Ponty, M. (1964a). Signs. S.1.:Northwestern University press.

Merleau-Ponty, M. (1964b). The Primacy of Perception. S.1.:Northwestern University press.

Merleau-Ponty, M. (1967). The structure of behavior. Beacon Press.

Merleau-Ponty, M. (2012). Phenomenology of perception. Routledge.

Olive, L. J. (2014). Reflecting on the tensions between emic and etic perspectives in life history research: Lessons learned. Forum Qualitative Sozialforschung / Forum: Qualitative Social Research, 15(2).

Oliver, D. G., Serovich, J. M., \& Mason, T. L. (2005). Constraints and opportunities with interview transcription: Towards reflection in qualitative research. Social Forces, 82(2), 1273-1289.

Osler, L. (2020). Feeling togetherness online: A phenomenological sketch of online communal experiences. Philosophy of Cognitive Sciences, 19, 569-588.

Park, H. S. et al. (2019). Falling for a fake: The role of kinematic and non-kinematic information in deception detection. Perception, $0(0)$, pp. 1-8. 
Pei, A. (2019). This esports giant draws in more viewers than the Super Bowl, and it's expected to get even bigger. [online] available at: https://www.cnbc.com/2019/04/14/league-of-legends-gets-more-viewersthan-super-bowlwhats-coming-next.html [accessed 202005 27].

Ravn, S. (2016). Phenomenological analysis in sport and exercise. In Routledge Handbook of Qualitative Research in Sports and Exercise (pp. 206-218). Routledge.

Ravn, S., \& Hansen, H. P. (2013). How to explore dancers' sense experiences? A study of how multi-sited fieldwork and phenomenology can be combined. Qualitative Research in Sport, Exercise and Health, pp. $196-213$.

Ravn, S., \& Høffding, S. (2017). The promise of 'sporting bodies' in phenomenological thinking : How exceptional cases of practice can contribute to develop foundational phenomenological concepts. Qualitative Research in Sport, Exercise and Health, 9(1), 56-68.

Rosa, H. (2019). Resonance. 1st ed. Cambridge: Polity.

Sebanz, N., \& Schiffrar, M. (2009). Detecting deception in a bluffing body: The role of expertise. Psychonomic Bulletin and Review, 16(1), 170-175.

Slater, M. (2009). Place illusion and plausibility can lead to realistic behavior in immersive virtual environments. Philosophical Transactions of the Royal Society B, 364, 3549-3557.

Smith, M. J., Birch, P., \& Bright, D. (2019). Identifying stressors and coping strategies of elite Esports competitors. International Journal of Gaming and Computer-Mediated Simulations, 11(2), 22-39.

Stake, R. E. (1995). The art of case study research. SAGE Publications.

Steed, A., Friston, S., \& Lopez, M. M. (2016). An 'in the wild' experiment on presence and embodiment using consumer virtual reality equipment. IEEE Transactions on Visualization and Computer Graphics, 22(4), $1406-1414$.

Tanaka, S. (2015). Intercorporeality as a theory of social cognition. Theory and Psychology, 25(4), 455-472.

Taylor, T., \& Witkowski, E. (2010). This is how we play it: What a mega-LAN can teach us about games. Proceedings of the fifth international conference on the foundations of digital games, pp. 195-202.

Tietz, A. (2015). Embodiment online and interaction in massively multiplayer online games. Contributions from European Symbolic Interactionists: Conflict and Cooperation, pp. 119-136.

Turkle, S. (2017). Alone together: Why we expect more from technology and less from each other. Hachette UK.

Walsh, P. J. (2020). Intercorporeity and the first-person plural in Merleau-Ponty. Continental Philosophy Review, 53, 21-47.

Witkowski, E. (2012). On the digital playing field: How we "do sport" with networked computer games. Games and Culture, pp. 349-374.

Publisher's note Springer Nature remains neutral with regard to jurisdictional claims in published maps and institutional affiliations.

\section{Affiliations}

\section{David Ekdahl $^{1} \cdot$ Susanne Ravn ${ }^{1}$}

1 Department of Sports Science and Clinical Biomechanics (Research Unit: Movement, Culture and Society / MoCS), University of Southern Denmark, Campusvej 55, 5230 Odense M, Denmark 